\title{
Impaired expression of the COSMOC/MOCOS gene unit in ASD patient stem cells
}

\author{
Pauline Rontani ${ }^{1} \cdot$ Olivier Perche $^{2,3} \cdot$ Louise Greetham $^{1} \cdot$ Nicolas Jullien $^{1} \cdot$ Bruno Gepner $^{1} \cdot$ François Féron $^{1}$. \\ Emmanuel Nivet ${ }^{1} \cdot$ Madeleine Erard-Garcia $^{1,2}$
}

Received: 13 July 2019 / Revised: 17 March 2020 / Accepted: 3 April 2020 / Published online: 23 April 2020

(c) The Author(s) 2020. This article is published with open access

\begin{abstract}
Autism spectrum disorders (ASD) are complex neurodevelopmental disorders with a very large number of risk loci detected in the genome. However, at best, each of them explains rare cases, the majority being idiopathic. Genomic data on ASD derive mostly from post-mortem brain analyses or cell lines derived from blood or patient-specific induced pluripotent stem cells (iPSCS). Therefore, the transcriptional and regulatory architecture of the nervous system, particularly during early developmental periods, remains highly incomplete. To access the critical disturbances that may have occurred during pregnancy or early childhood, we recently isolated stem cells from the nasal cavity of anesthetized patients diagnosed for ASD and compared them to stem cells from gender-matched control individuals without neuropsychiatric disorders. This allowed us to discover MOCOS, a non-mutated molybdenum cofactor sulfurase-coding gene that was under-expressed in the stem cells of most ASD patients of our cohort, disturbing redox homeostasis and synaptogenesis. We now report that a divergent transcription upstream of MOCOS generates an antisense long noncoding RNA, to which we coined the name COSMOC. Surprisingly, COSMOC is strongly under-expressed in all ASD patients of our cohort with the exception of a patient affected by Asperger syndrome. Knockdown studies indicate that loss of COSMOC reduces MOCOS expression, destabilizes lipid and energy metabolisms of stem cells, but also affects neuronal maturation and splicing of synaptic genes. Impaired expression of the COSMOC/MOCOS bidirectional unit might shed new lights on the origins of ASD that could be of importance for future translational studies.
\end{abstract}

\section{Introduction}

ASD are a heterogeneous group of neurodevelopmental disorders characterized by persistent deficits in social communication and interactions as well as restrictive, repetitive patterns of behaviors (DSM-5). So far, the precise mechanisms that underlie the complex pathophysiology of ASD remain obscure, which is largely due to

Supplementary information The online version of this article (https:// doi.org/10.1038/s41380-020-0728-2) contains supplementary material, which is available to authorized users.

Madeleine Erard-Garcia

madeleine.erard@cnrs-orleans.fr

1 Aix Marseille University, CNRS, INP, UMR 7051 Marseille, France

2 Orléans University, CNRS, INEM, UMR 7355 Orleans, France

3 Department of Genetics, Regional Hospital, Orleans, France the heterogeneous phenotypic presentation of the disease and the complexity of its inheritance. The genetic heterogeneity associated to ASD underscores the importance of identifying convergent pathways and molecular mechanisms that are responsible for these disorders. So far, collaborative studies between professionals/researchers and autistic adults together with shared resources suggest that over than 1000 genes are involved in ASD susceptibility [1-3]. However, at best, they explain less than $10 \%$ of the cases and the vast majority of ASD cases are idiopathic. The known functions of many of those candidate genes led the researchers to associate autism with an abnormal synaptic plasticity and so perturbations on neuronal/synaptic homeostasis [4, 5]. Although ASD cannot be reduced to neuronal and synaptic dysfunctions, it indicates that the identification of rare variants that could explain such disturbances during early development is important.

With the aim of identifying genes likely to contribute to the initial events leading to ASD etiology, we previously 
used olfactory stem cells (OSCs) isolated from nasal biopsies of ASD patients. These cells, displaying multipotent features [6, 7], are representative of early stages of ontogenesis and allow us to search for altered gene expression not observed in other cell types. Using pangenomic DNA microarray, we recently reported an under-expression of a transcript coding for the enzyme molybdenum cofactor sulfurase (MOCOS) in most ASD patients of our cohort [8]. MOCOS is mainly known for adding a sulphur on MOCO, a cofactor necessary for two enzymes - xanthine oxidoreductase (XOR) and aldehyde oxidase (AOX) - involved in purines metabolism [9]. We observed that reduced expression of MOCOS induces a hypersensitivity to oxidative stress, a perturbed synaptogenesis and an abnormal neurotransmission [8], features associated to neurodevelopmental deficits [4, 5, 10]. Given that MOCOS is involved in multiple biological and neurobiological functions, its loss of function is therefore coherent with phenotypic and aetiopathogenic heterogeneity in the ASD population.

In this study, we expand on that pioneer work and now report on the analysis of the upstream regulators of MOCOS. Recent advances in transcriptome sequencing have revealed long noncoding RNAs (lncRNAs) as a new class of genes able to regulate major biological processes, including synaptic development, maturation and plasticity [11-13]. However, understanding how IncRNAs control brain development and function remains elusive and no individual variant has been robustly associated to ASD so far [14-17]. With the help of frontline genetic tools, we have discovered that the promoter region of MOCOS can be read bidirectionally and induces the transcription of both MOCOS and an antisense IncRNA, which we named COSMOC. Antisense lncRNAs, usually poorly expressed and not conserved during evolution, were long considered as background transcripts. Nevertheless, we now know that they regulate almost all stages of gene expression, at the pre-transcriptional, transcriptional and posttranscriptional levels. LncRNAs can act in trans, on genes located on distant loci or other chromosomes, and in cis, on proximally positioned genes [18]. Therefore, COSMOC may be an interesting potential regulator of genes that could have critical roles in ASD.

To assess the possible implication of COSMOC on MOCOS expression and, more widely, its roles on cell metabolism, we used our initial model, namely human OSCs. We observed that (i) COSMOC transcription is necessary for the regulatory activity of this locus; and (ii) 10 out of 11 ASD patients display a drastic reduction of COSMOC expression, while an Asperger patient was only slightly perturbed. Then, we analyzed transcript variations when COSMOC was silenced and, further down, highlighted perturbations in lipid biosynthesis, chromatin organization, energy metabolism and neuron maturation, as well as insults aggravating the neurological outcome of ASD patients. We thus unveiled hidden functions of COSMOC that support the possible role that this lncRNA can play in ASD.

\section{Material and methods}

\section{ASD patients and cell culture}

Human nasal olfactory stem cells (OSC) from 11 patients and 11 age- and gender-matched control individuals without diagnosed neuropsychiatric disorders were taken under general anesthesia as previously described [8]. Primary OSCs and iPSC lines, as well as SH-SY5Y, U138-MG, HepG2, HEK-293 and Caco-2 cell lines were cultured as described in Supplementary Information.

\section{Derivation of neural progenitor cells and neurons from iPSCs}

Neural progenitor cells (NPCs) were differentiated from human iPSCs (hiPSCs) by applying two different differentiation protocols as described in Supplementary Information. hiPSC-NPCs were differentiated into mature neurons with the BrainPhys ${ }^{\mathrm{TM}}$ Neuronal Medium Kit (Stem Cell Technologies) following manufacturer's instructions as specified in Supplementary Information.

\section{SH-SY5Y differentiation}

SH-SY5Y differentiation was performed as described in Supplementary Information. For knockdown experiments, undifferentiated cells were incubated during $24 \mathrm{~h}$ with siRNA before the initiation of differentiation. The differentiation was stop at day 5 .

\section{Generation of CRISPR-Cas9 mediated COSMOC- Knock-Out hiPSCs}

CRISPR sgRNAs were designed using Crispor design tool (http://crispor.tefor.net) and cloned into cAB03 as described in Supplementary Information. The generation of COSMOC-Knock-Out hiPSC lines is detailed in supplementary Information,

\section{Transfection of cells and primers}

Twenty-four hours after seeding, cells were transfected with Stealth RNAi ${ }^{\mathrm{TM}}$ siRNAs (Life Technologies) using standard protocols and primers listed in Supplementary Information and Supplementary Tables 1 and 2. 


\section{Microarray gene expression analysis}

OSC from two different healthy individuals were collected $48 \mathrm{~h}$ after treatment with siRNAs. Genome-wide transcriptional profiling was performed by Human Exon 1.0 ST arrays (Affymetrix) following manufacturer's instructions. Each condition was performed in duplicate. Microarray data analysis is detailed in Supplementary Information. The microarray data (GSE122434) can be accessed in the Gene Expression Omnibus (GEO) repository at the National Center for Biotechnology Information.

\section{RNA isolation and real-time PCR analysis}

RNA samples extracted from OSCs were tested with qRTPCR as specified in Supplementary Information.

\section{Western blot analysis}

Protein expression analyses in OSCs were performed using a standard protocol as described in Supplementary Information. Signals were visualized with ECL chemiluminescence kit (GE Healthcare) and quantified using ImageJ software.

\section{Oxidative stress induction, ROS assay and flow cytometry analysis}

Twenty-four hours after seeding, cells were stressed for 2 or $4 \mathrm{~h}$ in the presence of $\mathrm{H}_{2} \mathrm{O}_{2}(500 \mu \mathrm{M})$ before RNA extraction. Intracellular ROS was measured by staining with CellRox ${ }^{\circledR}$ Deep Red Flow Cytometry Assay kit (Life Technologies) using FacsCanto cytometer (Becton Dickinson). Data were analyzed using FACS DIVA software.

\section{Immunocytochemistry}

Cells were fixed in paraformaldehyde at room temperature and immunostainings were performed using standard procedures as described in Supplementary Information.

\section{Statistical evaluation}

Statistical analyses were performed by using standard unpaired Student's t-tests or one-way analysis of variance, and were carried out using Prism software (GraphPad Software, La Jolla, CA, USA) or Excel software (Microsoft, Redmond, WA, USA). All data are presented as mean \pm s.d. and represent a minimum of three independent experiments with at least three technical replicates. Estimate of variance was performed within each group of data being statistically compared. A value of $P<0.05$ was considered as statistically significant.

\section{Results}

\section{COSMOC, an antisense long noncoding RNA upstream of MOCOS}

As a first attempt to apprehend the transcriptional control of MOCOS expression, we knocked down several putative upstream regulators, as detected using MatInspector (Genomatix) and TFBind software, but none of them successfully deregulated MOCOS expression (Supplementary Fig. 1a-c). We then used the data generated by advanced genomic tools, such as ENCODE and FANTOM5 and identified a divergent transcription in the MOCOS promoter region. This GC-rich region (GC- content: 65\%) induces a transcription initiation on both sides with opposite orientations, generating an upstream antisense lncRNA near the 5'end of MOCOS, to which we coined the name COSMOC (Fig. 1a). COSMOC is a 609 nucleotides long and polyadenylated RNA (Supplementary Fig. 2a). Similarly to many other lncRNAs, it contains only two exons [19], separated by a long intron of $6845 \mathrm{bp}$ and the noncoding anti-sense initiation of COS$M O C$ is located $73 \mathrm{bp}$ upstream of the transcription starting site of MOCOS (Fig. 1a and Supplementary Fig. 2b). COSMOC has no evidence of coding potential as indicated by its absence in protein database, a low PhyloCSF score [20] and no open reading frame longer than 150 nucleotides. Consistent with a crosstalk between coding and noncoding transcripts arising from the same locus [21], COSMOC and MOCOS exhibit a broad and similar pattern of expression across different human cell lines and tissues (Fig. $1 \mathrm{~b}$ and Supplementary Fig. 2c). Of note, cell fractioning followed by qPCR analysis revealed that COSMOC can be found in the nucleus but it is predominantly located in the cytosol of OSCs (Fig. 1c), suggesting that its mode(s) of action on cellular mechanisms should not be reduced to a transcriptional regulator.

\section{COSMOC is under-expressed in ASD patient stem cells}

To assess the functions of COSMOC and determine whether it can regulate $M O C O S$ expression in a cis manner, we decided to use small interfering RNAs (siRNAs) to deplete both genes in human OSCs. When compared with cells transfected with scrambled small interfering RNAs (siControl), siRNA directed against COSMOC (si-COSMOC) significantly diminished MOCOS transcript (Fig. 1d) and the corresponding protein by almost $50 \%$ (Fig. 1e). In contrast, siRNA directed against MOCOS (si-MOCOS) did not affect $C O S M O C$ expression (Fig. 1f, g). Of note, this effect on MOCOS was found specific since COSMOC depletion did not alter the expression of $E L P 2$, its upstream neighbor gene (Supplementary Fig. 2b, d). 
a

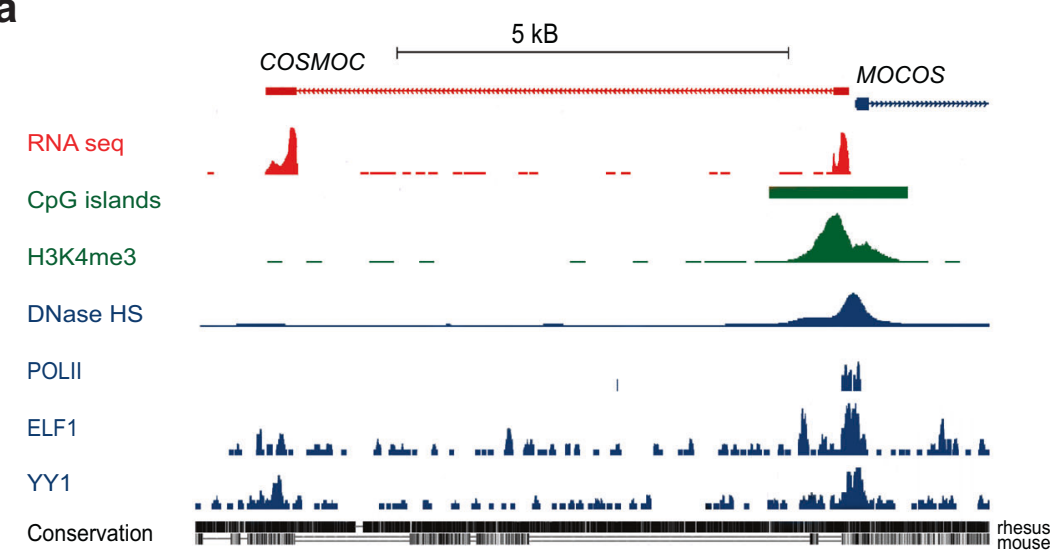

b

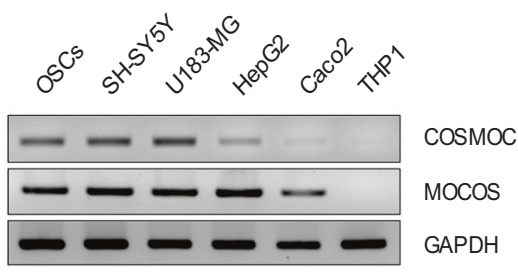

C

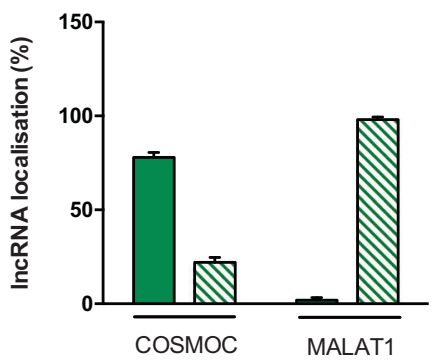

f

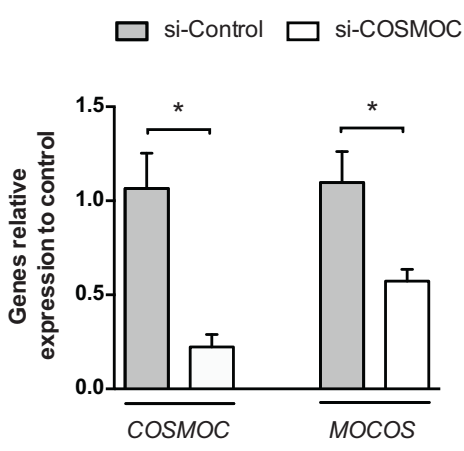

d si-Control $\square$ si-MOcos

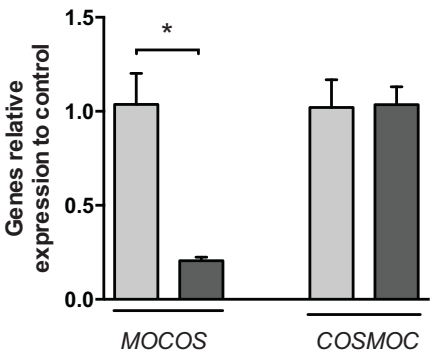

g

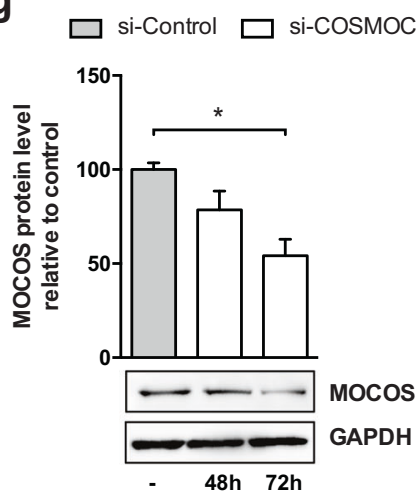

e

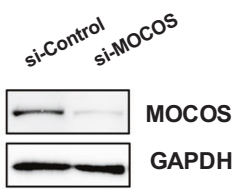

h

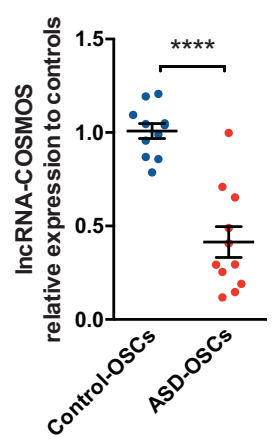

Fig. 1 The IncRNA COSMOC regulates its neighbor gene MOCOS and its dysregulation affects all ASD patients of our cohort. a UCSC genome browser image depicting divergent transcription in the promoter region of the human MOCOS gene. The lncRNA COSMOC (also called RP11-49111.1, transcript AC023043.1-201 or LOC101927809) spans $7454 \mathrm{bp}$ and comprises 2 exons of $214 \mathrm{bp}$ and $395 \mathrm{bp}$ separated by a long intron of $6845 \mathrm{bp}$. It is transcribed in antisense direction to MOCOS. Tracks showing RNA-seq, CpG islands, Histone $\mathrm{H} 3 \mathrm{~K} 4$ trimethylation (H3K4me3) sites, DNase hypersensitivity spick clusters (DNase HS), RNA polymerase II binding (POLII) and binding sites for ELF1 and YY1transcription factors are from the human neuroblastoma cell line SK-N-SH. Baselevel sequence comparison between COSMOC in human, monkey and mouse genomes indicates poor conservation in mice. b Agarose gel electrophoresis of COSMOC PCR products and Western blot analysis of MOCOS expression in a variety of cell lines. The specificity of the amplified products was confirmed by subsequent sequencing. GAPDH was used as a loading control for western blot. c Cell fractionation and qRT-PCR analysis of COSMOC expression in OSC. MALAT-1 is used as a control for nucleus/cytoplasm fractionation $(n=3)$. d qRTPCR analysis of COSMOC and MOCOS expression in response to MOCOS knockdown in OSCs. Cells were transfected with scrambled interfering RNAs (si-Control) or interfering RNAs directed against MOCOS (si-MOCOS) $(n=4$; Mann-Whitney test: $* p<0.05)$. e Western blot analysis of MOCOS expression in stem cells transfected with si-MOCOS. f qRT-PCR analysis of COSMOC and MOCOS expression in stem cells in response to COSMOC knockdown; $\left(n=5\right.$; Mann-Whitney test: $\left.{ }^{*} p<0.05\right)$. $\mathrm{g} 72 \mathrm{~h}$ after treatment of OSCs with si-COSMOC, MOCOS downregulation was revealed by western blot. h qRT-PCR analysis of COSMOC gene expression in OSCs of ASD patients (ASD-OSCs) and matched healthy individuals (Control-OSCs), $(n=11$; Mann-Whitney test: $* * * * p<0.0001)$. For all figures, $n$ stands for number of biological replicates of OSC. 
Considering our previous observations that revealed the deregulation of MOCOS in OSCs established from our cohort of ASD patients ( 8 ) we then measured the expression level of COSMOC in these same cells. Strikingly, we observed significant lower levels of COSMOC expression in all patients, an Asperger patient being the exception, when compared with their respective age- and gendermatch healthy controls (Fig. 1h). This data prompted us to study further COSMOC and identify how its downregulation could play a role in ASD pathology.

\section{COSMOC is involved in lipid metabolism, DNA regulation and redox homeostasis}

To start identifying putative roles of COSMOC, we performed a transcriptomic study on OSCs from two healthy individuals in which we knocked down COSMOC. We found a pool of 639 mis-expressed genes, of which 150 were upregulated and 477 downregulated (Fig. 2a and GEO database GSE122434). As expected, COSMOC-depleted OSCs cultures showed that MOCOS was downregulated (fold change: -1.36 and -1.86 in individual 1 and 2, respectively). Of higher interest, among the top misexpressed genes upon COSMOC depletion, eleven of them - ALDH9A1, DHCR7, ENPP2, FABP3, GLO1, GLUL, LIPA, MOCOS, PPT1, PTBP2, SEMA5A - have been previously associated with ASD (1-4; https://gene.sfari.org/). Furthermore, DAVID-based gene clustering analysis revealed two significantly distinct groups (Fig. 2b). The first cluster (enrichment score 3.21) included genes involved in lipid and sterol metabolism or cholesterol biosynthesis, whereas the second one (enrichment score 2.94) was composed of genes associated to nucleosome core, methylation and chromosome processes. Under the designation "Oxidation-reduction process", gene ontology classification indicated an altered detoxification machinery (Fig. 2b and Supplementary Table 3). Dysregulation of several ASDassociated genes and some genes involved in synaptic transmission or neural development were further validated by qPCR analyses (Fig. 2c-e). These data suggested that COSMOC modulates the functioning of relevant cellular mechanisms formerly implicated in ASD. Of note, MOCOS downregulation did not alter the panel of genes we tested, indicating that putative functional effects of COSMOC downregulation in ASD may act through MOCOSindependent mechanisms.

In light of our previous data showing an increased ROS production in ASD patient-derived OSCs, we next decided to measure the number of stressed cells in COSMOCsilenced conditions. We observed a mean increase of $39.7 \%$ (Fig. 2f and Supplementary Fig. 3a-c) in the number of stressed cells from those cultures treated with si-COSMOC. Since depletion in MOCOS and MOCOS-dependent enzymes (i.e., AOX and XDH) induces ROS (Supplementary Fig. 3a), we next treated OSCs for various time durations with hydrogen peroxide $\left(\mathrm{H}_{2} \mathrm{O}_{2}\right)$, a potent catalyst of ROS. By doing so, we assessed whether the disturbed redox homeostasis was a consequence of COSMOC depletion or COSMOC-induced MOCOS exhaustion. As shown in Fig. 2g, cells started transcribing COSMOC before MOCOS, suggesting that, within the COSMOC/MOCOS duo, $C O S M O C$ is the first to respond to oxidative stress.

\section{Modulated expression of COSMOC during neural differentiation}

LncRNAs being known as key regulators of neural differentiation [11,22], and ASD being primarily associated with neurodevelopmental disorders, we next examined whether COSMOC modulation could impact on neurodevelopmentallike processes by using in vitro strategies. First, and to test its potential role during neuronal differentiation/maturation we analyzed the dynamic expression of COSMOC in SH-SY5Y, a human neuroblastoma cell line able to differentiate into a homogeneous population of neuron-like cells [23]. As shown in Fig. 3a-c, SH-SY5Y cells exhibited a rapid and progressive increase of COSMOC expression during the differentiation procedure, reaching an 8-fold increase in 9-day old differentiated neuron-like cells. In parallel, MOCOS expression did not change throughout the differentiation. Of importance, and validating the acquisition of a post-mitotic phenotype in those differentiated cells, we observed an inversed correlation between $C D K 1$ expression and $M A P T$ expression (Fig. 3b), with CDK1 being involved in cell cycle whereas MAPT is critical for neuronal maturation. Neuronal differentiation was further confirmed by the acquisition of morphological changes resembling neuronallike morphology but also by the expression of the neuronal marker MAP2 as detected by immunocytochemical analysis (Fig. 3c).

Considering our initial observations we next investigated the effects that COSMOC knockdown may have on $\mathrm{SH}-$ SY5Y-based neuronal differentiation (Fig. 3d and Supplementary Fig. S4a-g). During the first 5 days of differentiation, no change was observed in COSMOC-downregulated differentiating neurons when compared with scrambled-siRNA treated ones (Fig. 3e-g). However, from day 6 of differentiation and onward, COSMOC knockdown caused morphological changes including cell shrinkage. Because these changes are important features of programmed cell death in neurons, we scored alterations in nuclear morphology and caspase 3 activation (Fig. 3h-k). At Day 5, we observed a significant higher number of apoptotic nuclei in COSMOC-downregulated neuronal-like cells, with a condensed and fragmented chromatin (Fig. 3h, i) while cell death was confirmed by a substantial increase in 
a

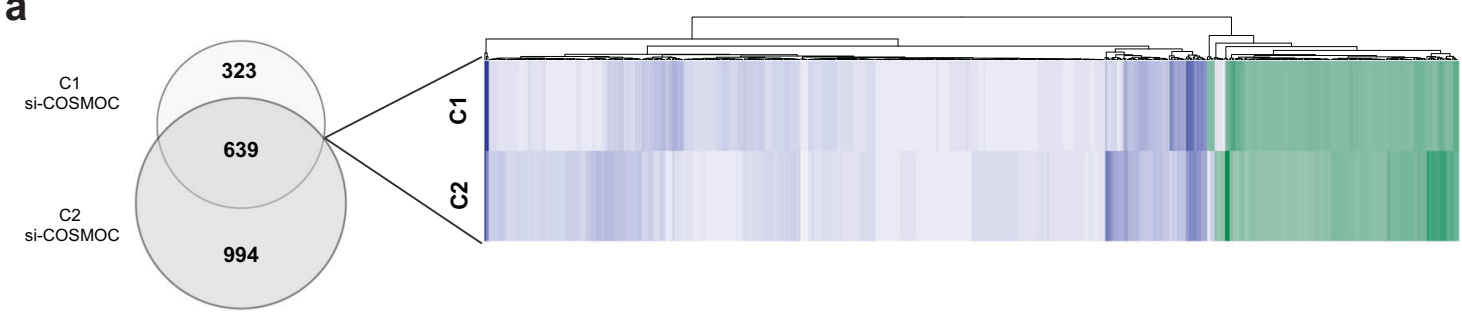

b

Gene count $\square$ Fold Enrichment

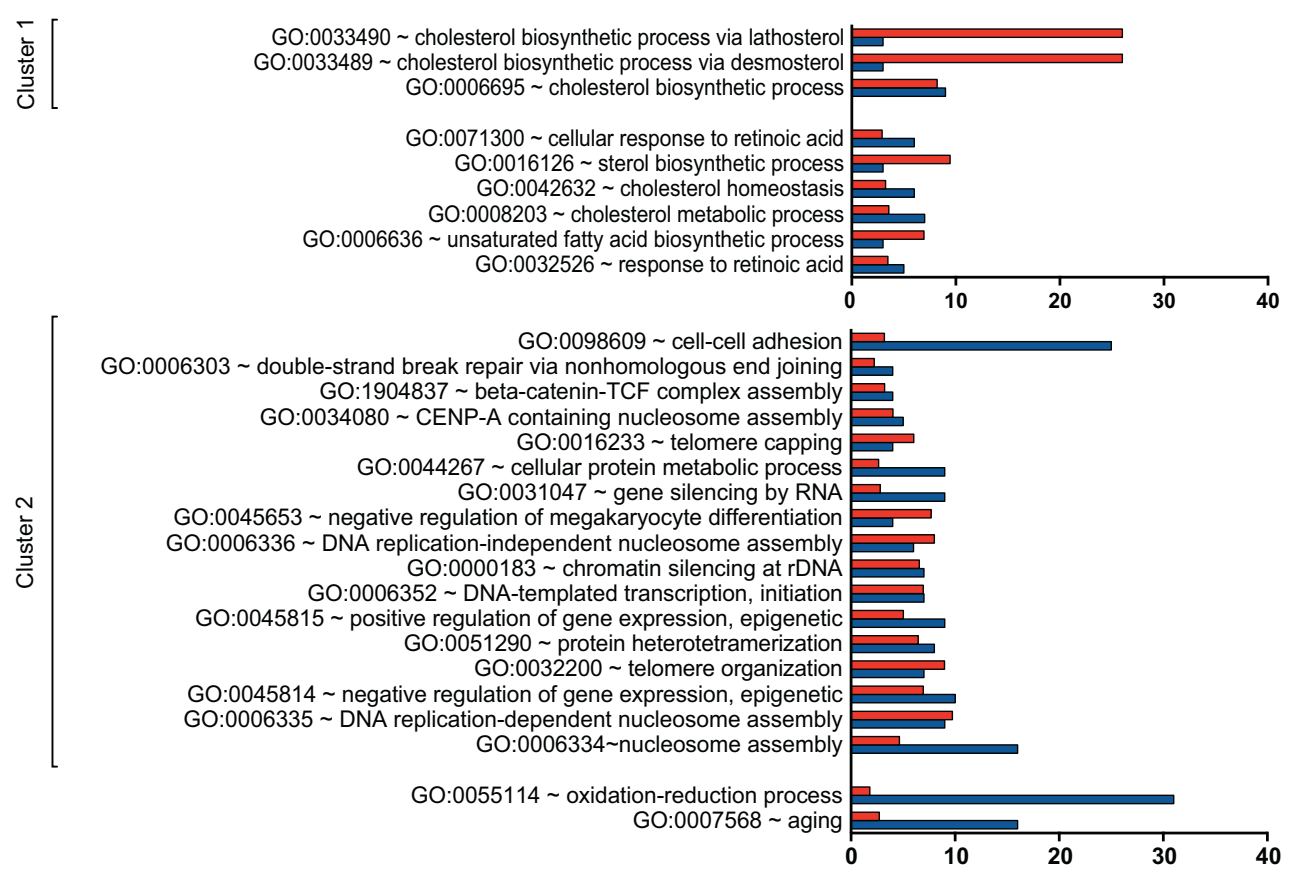

C

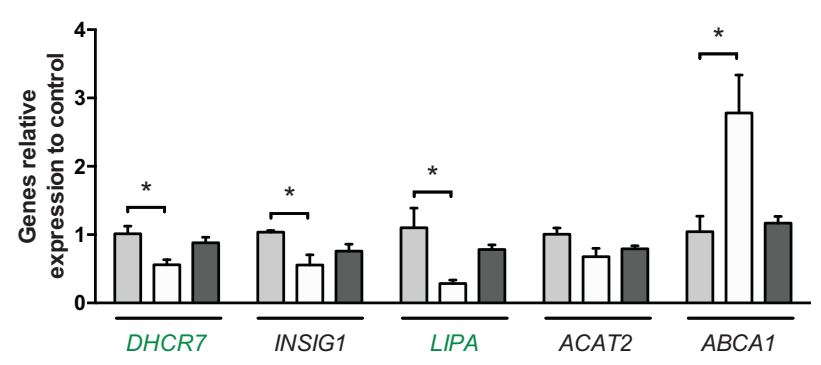

e

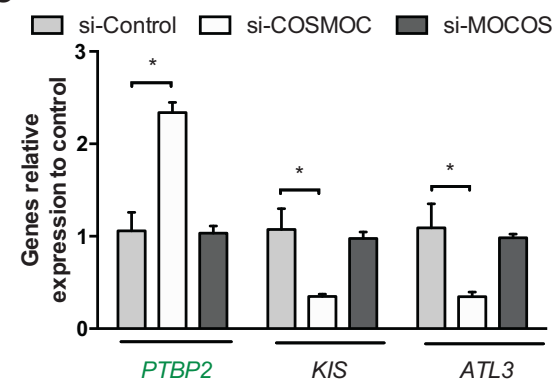

d

$\square$ si-Control $\square$ si-COSMOC $\square$ si-MOCOS

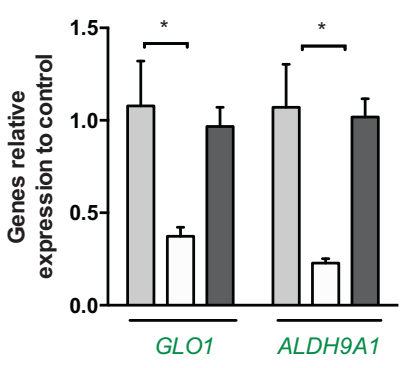

g

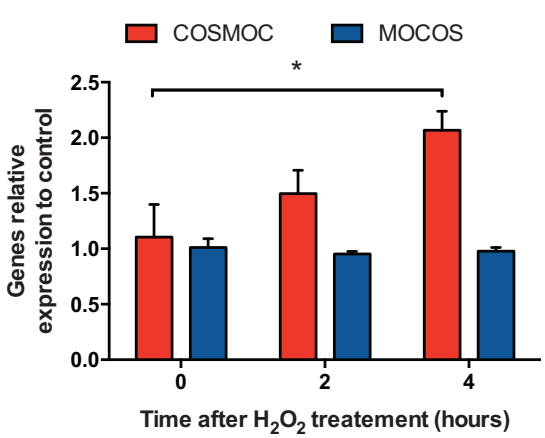


Fig. 2 COSMOC knockdown alters lipid metabolism, DNA regulation and redox homeostasis. a Venn diagram summarizing the number of genes differentially expressed in OSCs of control subjects $\mathrm{C} 1$ et $\mathrm{C} 2$ transfected with si-COSMOC after normalization on control OSCs $(n=2)$. A two dimensional representation of the common dysregulated genes is visible in the heat map. b Gene ontology (GO) terms significantly enriched among differentially expressed genes in COSMOC-depleted cells. Red bars indicate the fold enrichment of the Gene Ontology (GO) and blue bars indicate the gene count. Two enriched clusters respectively enriched with genes involved in lipid and sterol metabolism or cholesterol biosynthesis and genes involved in nucleosome core, methylation and chromosome process were detected. The GO terms oxidation-reduction process and aging are not clustered or significantly enriched but represent a significant number of deregulated genes. c-e RT-qPCR validation of the transcriptome analysis. Dysregulation of a subset of genes involved in cholesterol biosynthesis process (DHCR7 INSIG1, LIPA, ACAT2 and ABCA1), redox homeostasis (GLO1 and ALDH9A1) and synaptic transmission or neural development (PTBP2, KIS and ATL3) was confirmed in OSCs deficient in COSMOC compared with cells treated with siRNA control. Highly similar results were obtained with both microarray and RT-qPCR techniques $(n=4$; Mann-Whitney test: $* p<0.05)$. Genes known to be associated with ASD are written in green. $\mathbf{f}$ Quantitative analysis of oxidative stress in stem cells depleted in COSMOC, MOCOS, AOX, or XDH and comparison with cells treated with siControl $\left(n=4\right.$; Mann-Whitney test: $\left.{ }^{*} p<0.05\right)$. g RT-qPCR analysis of $C O S M O C$ and MOCOS expression after exposure of stem cells to $\mathrm{H}_{2} \mathrm{O}_{2}$ for 2 or $4 \mathrm{~h}\left(n=4\right.$; Mann-Whitney test: $\left.{ }^{*} p<0.05\right)$. For all figures, $n$ stands for number of biological replicates of OSC.

active caspase-3 (Fig. 3j, k). Of note, despite a slight increase in the number of apoptotic bodies, no significant impact on neuronal cell death was observed upon MOCOS downregulation (Fig. 3i), suggesting that COSMOC may act on neuronal cells in a MOCOS-independent manner. In that regard, it is worth noting that, as specified in our previous report, all-except-one ASD patients of our cohort are suffering from a loss of brain volume, atrophy or dysmorphy [8]. These results indicate that COSMOC could be a critical player in processes controlling neuronal survival and may participate to neurodevelopmental processes.

Therefore, in view of the fact that ASD pathogeny is perceived as a neurodevelopmental disorder that takes place during the initial steps of brain development, we asked whether COSMOC might be able to interfere with early developmental processes. Using the human induced pluripotent stem cells (hiPSC) technology, we first investigated the dynamic expression pattern of COSMOC during differentiation into neural progenitor cells (NPC). Interestingly, using two distinct induction protocols, we observed a burst of COSMOC expression as early as day 1 after neural induction of hiPSCs. This moderate increase was followed by a rapid but progressive decline during the next few days of differentiation until COSMOC expression became undetectable in differentiated NPCs (Supplementary Fig. 5a, b). The efficacy of our differentiation strategies was confirmed by PAX6, SOX2 and NESTIN immunostaining in hiPSC- derived NPCs and TUJ-1 and MAP2 staining in
NPC-derived differentiated neurons (Supplementary Fig. 4c).

In order to trace the effects that COSMOC knockout could have on the transition from pluripotency to neural/neuronal stages, we next used the CRISPR/Cas9 technology and successfully derived two different knockout $\left(\mathrm{COSMOC}^{-l-}\right)$ hiPSC lines (Supplementary Fig. 5d, e). $\mathrm{COSMOC}^{-/-}$hiPSCs and their respective $\mathrm{COSMOC}^{+/+}$ isogenic control line were differentiated into NPCs and we examined the expression of a small subset of neural progenitor associated genes (i.e., VIMENTIN, SOX2, NESTIN). Overall, Supplementary Fig. $5 \mathrm{f}-\mathrm{h}$ show that the selected genes followed a similar trend of expression upon neural induction, which indicates that knocking-out COSMOC is not sufficient to impair the generation of neural progenitors. However, we cannot rule out the possibility that those NPCs are not phenotypically identic to the ones derived from $\mathrm{COSMOC}^{+/+}$iPSCs.

\section{COSMOC impair synaptogenesis via PTBP2, a polypyrimidine tract binding protein}

To determine potential changes resulting from COSMOC downregulation during SH-SY5Y differentiation, we examined the dynamic transcription pattern of genes selected from the aforementioned microarray data. Interestingly, a significant increase of $P T B P 2$ expression was observed throughout the time course of differentiation of SH-SY5Y depleted for COSMOC (Supplementary Fig. 6). Since the introduction of a premature termination codon in $P T B P 2$ transcripts results in their degradation via nonsensemediated decay (NMD) [24], we examined the ratio of inclusion/exclusion of exon 10 (unspliced/spliced mRNA) in undifferentiated versus differentiated COSMOC-downregulated SH-SY5Y. Figure 4a shows a significant increase of PTBP2 exon 10 inclusion and Fig. 4b, c indicate a nearly $50 \%$ increased expression of PTBP2 protein level when siCOSMOC-treated cells were compared with control cells at day 5 of differentiation. In a similar way, a substantial rise of exon 10 inclusion was noticed in COSMOC-targeted OSCs (Supplementary Figure 6a). Of note, PTBP2 was never disturbed in si-MOCOS treated cells (Fig. 2e, Fig. 4a-c and Supplementary Fig. 6a) and PTBP1 expression, a splicing regulator of PTBP2 [24], did not changed during neuronal differentiation of si-COSMOC-treated cells (Supplementary Fig. 6b). These data demonstrate that COSMOC downregulation induces the increase of PTBP2. This may occur directly or indirectly by either impairing PTBP2 autoregulation or disturbing the trans-splicing activity of its upstream RNA-binding regulators.

Of major interest, PTBP2 controls a genetic program crucial for neuronal maturation that regulates a variety of target genes including GABA receptors but also Gephyrin 
a

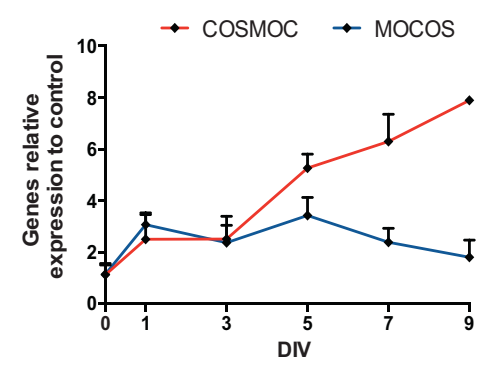

b

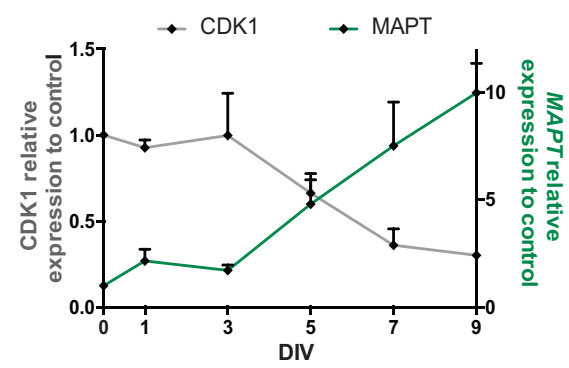

d

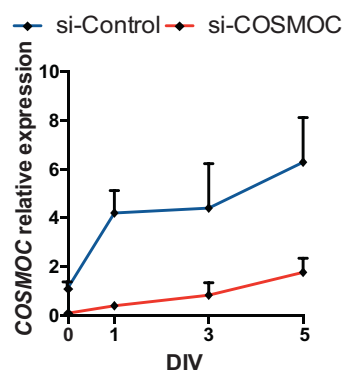

e

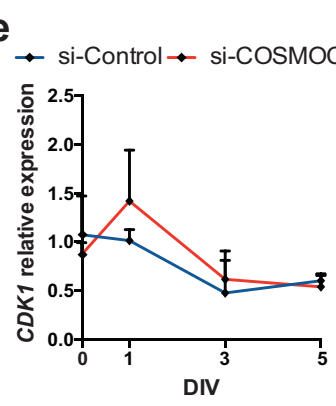

h

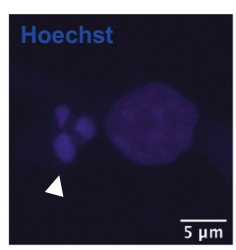

f

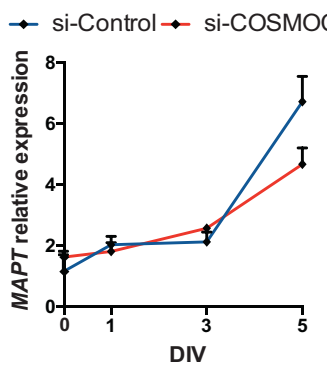

C

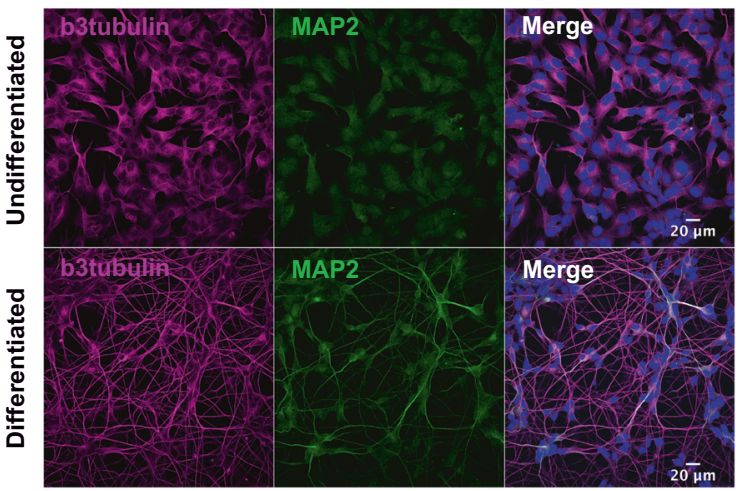

g

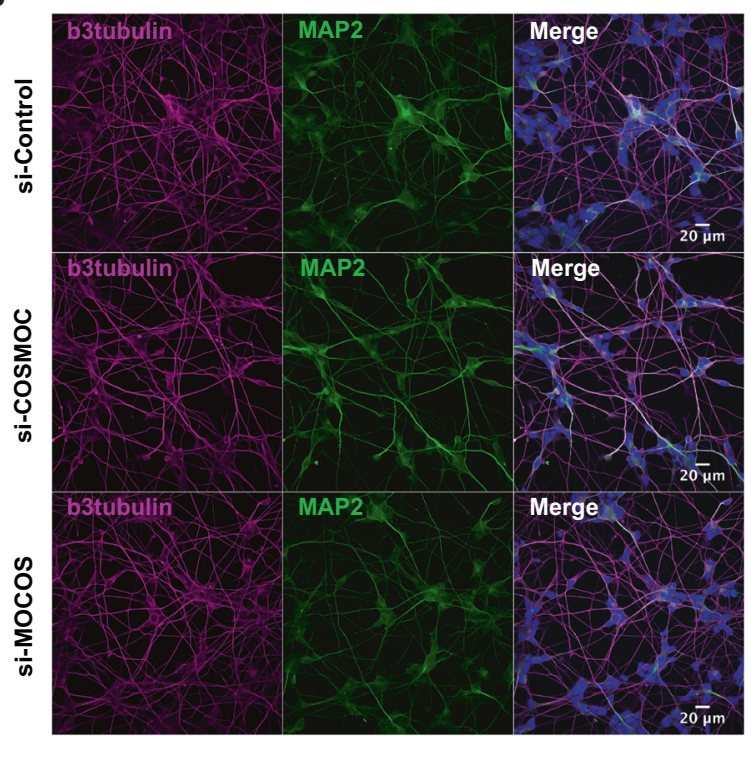

and PSD95 (Postsynaptic Density Protein 95), representing pivotal proteins for synaptic maturation and plasticity $[25,26]$. Since PTBP2 may inhibit the inclusion of PSD95 exon 18 [24], we decided to assess exon 18 splicing in COSMOC-downregulated SH-SY5Y cells ongoing differentiation. As shown on Fig. 4d, although no difference was 
Fig. 3 COSMOC is required for neuronal survival. a RT-qPCR analysis of COSMOC and MOCOS expression during neuronal differentiation of SH-SY5Y cells for 9 days in vitro (DIV). Gene expression was normalized to time-point $0(n=3)$. b Neuronal differentiation was confirmed by RT-qPCR analysis showing decreased expression of the cell division marker $C D K 1$ and increased expression of the neuronal marker MAPT. Data were normalized to time-point 0 $(n=3)$. c Immunocytochemistry with the neuronal differentiation markers $\beta$ III tubulin and MAP2 on undifferentiatied and differentiated SH-SY5Y cells at 9 days. d RT-qPCR analysis showing effective depletion of COSMOC in SH-SY5Y cells during the first 5 days of neuronal differentiation. SH-SY5Y cells treated with si-COSMOC were compared with cells incubated with scrambled siRNA and data were normalized to time-point $0(n=3)$. e, $\mathbf{f}$ RT-qPCR confirmation of effective neuronal differentiation of SH-SY5Y depleted in COSMOC expression during the first 5 DIV. The expected inverse correlation between $C D K 1$ expression (e) and MAPT expression (f) was observed in SH-SY5Y treated with si-COSMOC and those treated with scrambled siRNA. Data were normalized to time-point $0(n=3)$. g Immunocytochemistry showing $\beta \mathrm{III}$ tubulin and MAP2 staining of SH-SY5Y cells treated with siCOSMOC, siMOCOS or control siRNA at 5 DIV. h Analysis of cell death by nuclear staining with Hoechst. Example of a nucleus counted as apoptotic after staining with Hoechst (leftmost) and compared with two adjacent normal nuclei. i Quantification of apoptotic nuclei at 5 DIV in SH-SY5Y knockdown for COSMOC, MOCOS or control siRNA ( $n=4$; Mann-Whitney test: $* p<0.05)$. Representative Western blot $(\mathbf{j})$ and quantification $(\mathbf{k})$ of cleaved products of caspase-3 (17 and $19 \mathrm{KDa}$ ) at $5 \mathrm{DIV}$ in SH-SY5Y cells depleted in COSMOC, MOCOS or control siRNA. GAPDH was used as control. $(n=4$; Mann-Whitney test: $* p<0.05)$. Data are represented as mean \pm SEM. For all figures, $n$ stands for number of biological replicates of SH-SY5Y cells.

observed in undifferentiated cell, knocking down COSMOC significantly decreased exon 18 inclusion (about 40\%) in differentiated cells at Day 5. Altogether, these data support the notion that $C O S M O C$ deregulation may impair synapse development. Conversely, MOCOS knockdown failed to i) interfere with normal development of SH-SY5Y cells and ii) perturb COSMOC potential targets, supporting further an uncoordinated regulation of the transcriptional bidirectional unit, during neuronal differentiation (Fig. 4a-d, Supplementary Fig. 6a).

Taking this into account, and leveraging on our COS$\mathrm{MOC}^{-1-}$ iPSC-derived NPC model, we sought for possible alteration of PSD95 expression in $\mathrm{COSMOC}^{-1-}$ NPCs ongoing neuronal maturation. We found that PSD95 expression was rapidly detectable in $\mathrm{COSMOC}^{+/+}$iPSCderived neurons and stable at the two different time points we analyzed (Day 7 and Day 14 of differentiation). Surprisingly, PSD95 expression was found unstable in COS$\mathrm{MOC}^{-1-}$ neurons ongoing differentiation, indicating that PSD95 was changing in a random manner across the differentiation. These data support a possible role of COSMOC in the regulation/stabilization of PSD95 during neuronal maturation, and the absence of COSMOC may lead to defects associated to PSD95 expression (Supplementary Fig. 4i, j).
Finally, we assessed $P T B P 2$ expression in ASD patients. We observed that the unspliced isoform of $P T B P 2$ was upregulated in all ASD patients' OSCs (Fig. 4e, f). These observations suggest that, in low COSMOC-expressing neuronal cells deregulation of PTBP2 and synapseassociated genes may alter neurotransmission and induce neurological disorders, including ASD.

\section{Discussion}

The current study, built on our previous data that highlighted the reduced expression of MOCOS as a risk factor for ASD [8], reports an enthralling finding: COSMOC, an upstream antisense lncRNA located in the promoter region of MOCOS, is under-expressed in nearly all ASD patients of our cohort, the only exception being a patient affected by Asperger syndrome. Such a finding indicates that the deregulation of the COSMOC/MOCOS duet might be considered as a biomarker for ASD.

Although a set of candidate ASD-associated lncRNAs have been identified in co-expression network analysis [27], complex bidirectional transcription units have not been probed and divergent gene pairs still need to be carefully defined and characterized to help elucidate the genetic pathogenesis of ASD. It is therefore essential to further study the complex mechanisms of action of the COSMOC/ $M O C O S$ gene pair in the overall neurodevelopmental processes. However, while several physiological functions have been assigned to MOCOS, nothing was previously known about COSMOC. We show here that COSMOC and MOCOS display a similar pattern of expression. Nonetheless, the pair relationships are not fully bi-directional: COSMOC depletion induces a $50 \%$ reduced expression of $M O C O S$ in OSCs but the reverse is not true. Although further research is needed to definitely exclude the presence of enhancer-like elements in the MOCOS promoter region and establish open chromatin state on this locus, our results indicate that COSMOC is finely tuned, could be a critical player in processes controlling neuronal survival and participate to neurodevelopmental processes.

At that point, our experimental approaches and results do not allow us to clearly define the mechanism(s) by which COSMOC regulates MOCOS. Actually, COSMOC function(s) may heavily depend on its subcellular localization and the adoption of specific structural modules with interacting partners [28]. In the nucleus, COSMOC may take part in the nuclear organization, mediating epigenetic changes to specific genomic loci and affecting the expression of its neighboring MOCOS transcripts in cis by recruiting transcription factors, RNA-binding proteins and chromatin-remodeling machineries to the site of transcription. This locus-specific environment could facilitate 
a

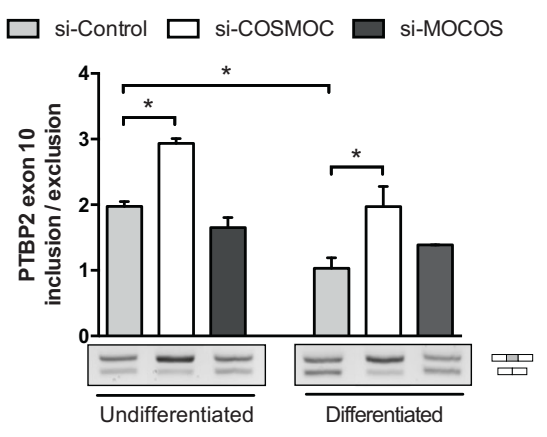

d

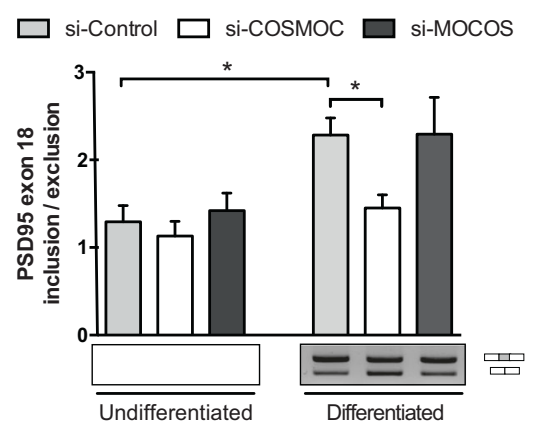

b

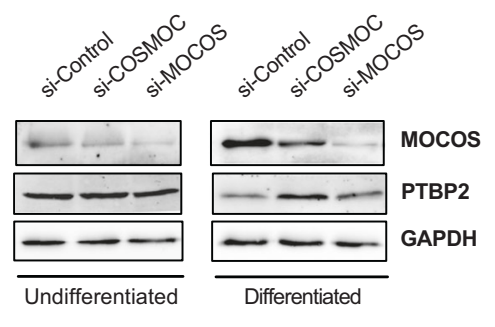

e

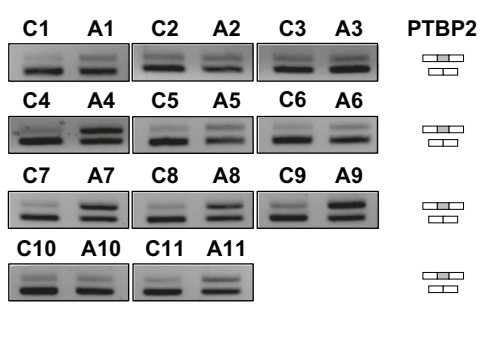

C
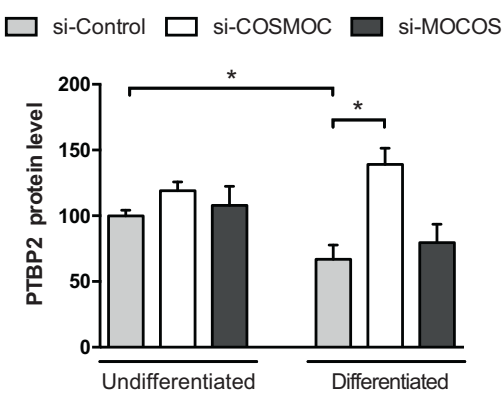

f

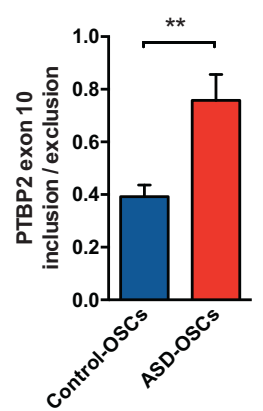

Fig. 4 COSMOC downreguation alters PTBP2 splicing in SHSY5Y and stem cells from ASD patients. a Semi-quantitative RTPCR analysis of PTBP 2 exon 10 splicing in undifferentiated or differentiated SH-SY5Y cells transfected with siRNAs $(n=4$; Mann-Whitney test: $\left.{ }^{*} p<0.05\right)$. b Representative western blot of MOCOS and PTBP2 expression and $\mathbf{c}$ quantification of PTBP2 protein level at 5DIV in undifferentiated and differentiated neuroblastoma cells. Cells were depleted in COSMOC or MOCOS expression and compared with cells treated with control siRNA. GAPDH was used as control. ( $n=4$; Mann-Whitney test: $* p<0.05)$. d Semi-quantitative

establishment of proper chromatin architecture to regulate MOCOS expression. Such a mechanism would be in agreement with our results and several reports investigating GC-rich regions and lncRNA functions [9, 29]. In the same way, the preferential localization and the role(s) of COSMOC in the cytoplasm are not completely resolved but modulation of key steps in post-transcriptional regulation of gene expression from mRNA processing to mRNA splicing, stability and translation or storage could also depend on cytoplasmic COSMOC.

In order to get a first overall picture of COSMOC primary and secondary targets, we performed a transcriptomic study based on the silencing of this lncRNA in OSCs. An impressive list of 639 genes is now associated to COSMOC depletion in OSCs, which could probably be extended to many other cell types of an organism. Reassuringly, eleven of them - ALDH9A1, DHCR7, ENPP2, FABP3, GLO1, GLUL, LIPA, MOCOS, PPT1, PTBP2, SEMA5A - were previously associated to ASD. Of note, among COSMOC-
RT-PCR analysis of PSD95 exon 18 splicing in undifferentiated or differentiated SH-SY5Y cells treated with siRNAs and assessed at 5DIV. ( $n=4$; Mann-Whitney test: $* p<0.05)$. e Agarose gels and f quantification of RT-PCR products of PTBP2 exon 10 inclusion. RNA was isolated from OSCs of ASD patients (from A1 to A11) and OSCs of their matched healthy individuals (from C1 to C11) $(n=11$; Mann-Whitney test: $\left.{ }^{* *} p<0.01\right)$. Data are represented as mean \pm SEM. For all graphs, $n$ stands for the number of biological replicates of SH-SY5Y cells or OSC.

target genes, PTBP2 and SEMA5A, but also ATL3, KIS or $Y W H A H$ are required for neural development, not to mention, GLUL, PPT1, but also STX2, GNG12 and FLOT1, all involved in synaptic transmission in ASD (1-5; https://gene. sfari.org/). Interestingly, some genes (such as YWHAH and UHMK1) are genes with functional roles in neurodevelopment and other psychiatric diseases. These findings converge further to indicate that COSMOC could be part of the mechanism that regulates genes contributing to ASD. However, more works need to be done to define whether its deregulation is specific to ASD or could also be involved in other neurological disorders.

Interestingly, gene ontology indicates that COSMOCregulated genes are strongly associated to lipid and sterol metabolism or cholesterol biosynthesis. These findings are in line with the reported associations between ASD symptoms and disorders of cholesterol biosynthesis, steroid anomalies and high prevalence of vitamin D deficiency [30, 31]. Indeed, pregnenolone, a cholesterol derivative, 
enhances the activation of emotion-controlling neuro-circuits and potentially relieves ASD symptoms [32, 33]. The cerebral cholesterol is essential for synapse functioning, myelin growth and axon wrapping, during early development [34]. Moreover, cholesterol modulates receptor binding and release of neurotransmitters such as oxytocin, GABA, acetylcholine and serotonin [30]. It also triggers the production of neurosteroids, a class of powerful modulators of $\mathrm{GABA}_{\mathrm{A}}$ and glutamate receptors [35, 36]. Accordingly, it can be conjectured that COSMOC deregulation disturbs cholesterol biosynthesis and participates to ASD by altering neuronal physiology during development, when most cholesterol is derived from de novo synthesis by neurons and massive amounts of cholesterol are needed for growth $[31,36]$.

In addition, detailed inspection of the transcriptome revealed that the "Oxidation-reduction process" was presenting a significant number of deregulated genes (i.e., 46), indicating an altered detoxification circuitry. This process appeared to be particularly remarkable since we previously described (i) a disturbed redox homeostasis in stem cells of the ASD patients composing our cohort; and (ii) a greater susceptibility of MOCOS knockout animals to reactive oxygen species (ROS) [8]. Accordingly, we now show increased resting ROS production in stem cells treated with siRNA against COSMOC. These results are strengthened by the increased expression of PTBP2, a multifunctional splicing factor known to promote a switch in the expression of glycolytic enzymes [37]. Altogether, these data indicate that ASD patients of our cohort display metabolic disorders and suggest that COSMOC might modulate redox metabolism in stem cells. These findings are unsurprising as ASD are associated with impairments in basic physiological processes such as redox homeostasis. Increased levels of ROS, advanced glycation end products (AGEs) and lowered levels of antioxidants (such as glutathione peroxidase, superoxide dismutase, reduced glutathione) are indeed frequently reported in ASD children [10, 38]. Moreover, clinical trials have also reported an improved behavior in ASD patients receiving antioxidant therapy [39]. ROS are certainly essential regulators of multitudes of normal physiological processes in neurons including cognition and memory. Since synaptic activity is known to produce ROS, COSMOC under-expression through excessive ROS production may be associated with decreased performance in cognitive function in ASD patients. In that regard, the enhancer-like activity of COSMOC on the MOCOS gene may help us to understand some cellular functions of COSMOC. Hence, an unbalanced stress response may occur at least in part through inactivation of XDH and AOX, two MOCOS-dependent enzymes involved in purine metabolism and ROS production. Noticeably, alteration of sulfur metabolism and anomalies in purine metabolism have been reported in autism [40, 41] while anti-purinergic therapy was found to be effective in alleviating ASD symptoms [42].

The reason for the burst of COSMOC expression during the differentiation process of iPSC to neural progenitors has not been addressed yet. Recent studies have shown that stem cell fate is partially regulated by ROS, which mediates the redox state of cells as a secondary messenger [43]. Similarly, the progressive increase of COSMOC expression during differentiation of SH-SY5Y neuroblastoma cells may also be related to the profound changes in antioxidant defences that have been reported to occur during SHSY5Ydifferentiation to neuron-like phenotype [44]. Keeping in mind that COSMOC is involved in oxido-reduction processes, one could speculate that a transient expression of COSMOC may participate to the fate decision of stem cells. In addition, lipid metabolic pathways are important for neurogenesis and large amount of lipids is used to produce new membranes required upon proliferation and differentiation. Elucidating how COSMOC affects lipid metabolism and homeostasis may help unify our understanding of the complex autism disorders.

Our data also revealed the control of COSMOC on the splicing of PTBP2, a RNA-binding proteins essential in post-transcriptional regulatory events. This is of major interest as PTBP2 is primarily expressed in the brain and, plays a crucial role in a splicing program required for neuronal differentiation $[25,26]$. As a matter of fact, PTBP2 binds to intronic polypyrimidine clusters in pre-mRNA molecules and participates to the assembly of other splicingregulatory proteins. Its association with autism is based on two studies reporting indel and missense variants in ASD probands [4, 45]. Interestingly, beside PTBP2, key regulators of alternative splicing, including the RBFOX1 family of proteins and SR100 are linked to neurodevelopmental disorders, including autism [2, 15]. In addition, alterations in the noncoding transcriptome and splicing of activity-dependent neuronal genes were reported in the cortex of ASD patients [15]. Supporting this, our data indicate that PSD95 expression, an important regulator of synaptic structure and plasticity, is affected by COSMOC, which may probably mediate through PTBP2 splicing defects. Altogether, the action of COSMOC on PTBP2 may play a wider action on numerous key cellular processes.

ASD are highly heritable with a number of risk loci ranging from hundreds to $>1000$. However, at best, each of them explains less than $1 \%$ of the cases and the vast majority of ASD cases are idiopathic. We report here on the identification of a new IncRNA, COSMOC, and its underexpression in nearly all autistic patients of our cohort. Misexpression of COSMOC and its adjacent partner MOCOS that is also found deregulated in most (80\%) patients of our cohort [8], may be one of the common 
mechanisms contributing to numerous ASD cases and part of the dysfunctions observed in ASD. Collectively and/or separately, the COSMOC/MOCOS duo is involved in energy metabolism, neuronal maturation, synapse formation and neurotransmitter secretion, features strongly associated to neurodevelopmental disorders, including ASD. Its perturbed expression might as well trigger some of the ASD comorbid pathologies, notably the gastro-intestinal symptoms. Even though further studies are required to identify COSMOC interacting partners and the mechanisms underlying its action on the nervous system, this study reports on the first discovery of COSMOC and its importance to push forward investigation on its role in ASD.

Acknowledgements We gratefully acknowledge David Laurenceau (from the Genetic department of the Regional Hospital of Orleans) and Delphine Stephan (from INP of Marseille) for technical assistance. The study was financed by the CNRS, Orleans University, Aix-Marseille University and the European funding in Region Centre-Val de Loire (FEDER N ${ }^{\circ}$ 2016-00110366=BIO-TARGET 2015-2018 and FEDER $\mathrm{N}^{\circ}$ EX005756=BIO-TARGETII).

\section{Compliance with ethical standards}

Conflict of interest The authors declare that they have no conflict of interest.

Publisher's note Springer Nature remains neutral with regard to jurisdictional claims in published maps and institutional affiliations.

Open Access This article is licensed under a Creative Commons Attribution 4.0 International License, which permits use, sharing, adaptation, distribution and reproduction in any medium or format, as long as you give appropriate credit to the original author(s) and the source, provide a link to the Creative Commons license, and indicate if changes were made. The images or other third party material in this article are included in the article's Creative Commons license, unless indicated otherwise in a credit line to the material. If material is not included in the article's Creative Commons license and your intended use is not permitted by statutory regulation or exceeds the permitted use, you will need to obtain permission directly from the copyright holder. To view a copy of this license, visit http://creativecommons. org/licenses/by/4.0/.

\section{References}

1. de la Torre-Ubieta L, Won H, Stein JL, Geschwind DH. Advancing the understanding of autism disease mechanisms through genetics. Nat Med. 2016;22:345-61.

2. Quesnel-Vallieres M, Weatheritt RJ, Cordes SP, Blencowe BJ. Autism spectrum disorder: insights into convergent mechanisms from transcriptomics. Nat Rev Genet. 2019;20:51-63.

3. Geschwind DH, Flint J. Genetics and genomics of psychiatric disease. Science. 2015;349:1489-94.

4. De Rubeis S, He X, Goldberg AP, Poultney CS, Samocha K, Cicek AE, et al. Synaptic, transcriptional and chromatin genes disrupted in autism. Nature. 2014;515:209-15.

5. Bourgeron T. From the genetic architecture to synaptic plasticity in autism spectrum disorder. Nat Rev Neurosci. 2015;16:551-63.
6. Delorme B, Nivet E, Gaillard J, Haupl T, Ringe J, Deveze A, et al. The human nose harbors a niche of olfactory ectomesenchymal stem cells displaying neurogenic and osteogenic properties. Stem Cells Dev. 2010;19:853-66.

7. Murrell W, Feron F, Wetzig A, Cameron N, Splatt K, Belette B, et al. Multipotent stem cells from adult olfactory mucosa. Dev Dyn. 2005;233:496-515.

8. Feron F, Gepner B, Lacassagne E, Stephan D, Mesnage B, Blanchard MP, et al. Olfactory stem cells reveal MOCOS as a new player in autism spectrum disorders. Mol Psychiatry. 2016;21: $1215-24$.

9. Mendel RR, Kruse T. Cell biology of molybdenum in plants and humans. Biochim Biophys Acta. 2012;1823:1568-79.

10. Gu F, Chauhan V, Chauhan A. Glutathione redox imbalance in brain disorders. Curr Opin Clin Nutr Metab Care. 2015;18:89-95.

11. Guttman M, Donaghey J, Carey BW, Garber M, Grenier JK, Munson G, et al. lincRNAs act in the circuitry controlling pluripotency and differentiation. Nature. 2011;477:295-300.

12. Briggs JA, Wolvetang EJ, Mattick JS, Rinn JL, Barry G. Mechanisms of long non-coding RNAs in mammalian nervous system development, plasticity, disease, and evolution. Neuron. 2015;88:861-77.

13. Briz V, Restivo L, Pasciuto E, Juczewski K, Mercaldo V, Lo AC, et al. The non-coding RNA BC1 regulates experience-dependent structural plasticity and learning. Nat Commun. 2017;8:293.

14. Tang J, Yu Y, Yang W. Long noncoding RNA and its contribution to autism spectrum disorders. CNS Neurosci Ther. 2017;23:645-56.

15. Parikshak NN, Swarup V, Belgard TC, Irimia M, Ramaswami G, Gandal MJ, et al. Genome-wide changes in lncRNA, splicing, and regional gene expression patterns in autism. Nature. 2016;540: $423-7$.

16. Wang $\mathrm{Y}$, Zhao $\mathrm{X}, \mathrm{Ju} \mathrm{W}$, Flory $\mathrm{M}$, Zhong J, Jiang $\mathrm{S}$, et al. Genome-wide differential expression of synaptic long noncoding RNAs in autism spectrum disorder. Transl Psychiatry. 2015;5: e660.

17. Ziats MN, Rennert OM. Aberrant expression of long noncoding RNAs in autistic brain. J Mol Neurosci. 2013;49:589-93.

18. Kopp F, Mendell JT. Functional classification and experimental dissection of long noncoding RNAs. Cell. 2018;172:393-407.

19. Derrien T, Johnson R, Bussotti G, Tanzer A, Djebali S, Tilgner H, et al. The GENCODE v7 catalog of human long noncoding RNAs: analysis of their gene structure, evolution, and expression. Genome Res. 2012;22:1775-89.

20. Lin MF, Jungreis I, Kellis M. PhyloCSF: a comparative genomics method to distinguish protein coding and non-coding regions. Bioinforma (Oxf, Engl). 2011;27:i275-282.

21. Ponjavic J, Oliver PL, Lunter G, Ponting CP. Genomic and transcriptional co-localization of protein-coding and long noncoding RNA pairs in the developing brain. PLoS Genet. 2009;5: e1000617.

22. Ramos AD, Anderson RE, Liu SJ, Nowakowski TJ, Hong SJ, Gertz C, et al. The long noncoding RNA Pnky regulates neuronal differentiation of embryonic and postnatal neural stem cells. Cell Stem Cell. 2015;16:439-47.

23. Chiocchetti AG, Haslinger D, Stein JL, de la Torre-Ubieta L, Cocchi E, Rothamel T, et al. Transcriptomic signatures of neuronal differentiation and their association with risk genes for autism spectrum and related neuropsychiatric disorders. Transl Psychiatry. 2016;6:e864.

24. Boutz PL, Stoilov P, Li Q, Lin CH, Chawla G, Ostrow K, et al. A post-transcriptional regulatory switch in polypyrimidine tractbinding proteins reprograms alternative splicing in developing neurons. Genes Dev. 2007;21:1636-52. 
25. Li Q, Zheng S, Han A, Lin CH, Stoilov P, Fu XD, et al. The splicing regulator PTBP2 controls a program of embryonic splicing required for neuronal maturation. Elife. 2014;3:e01201.

26. Zheng S, Gray EE, Chawla G, Porse PT, O’Dell TJ, Black DL. PSD-95 is post-transcriptionally repressed during early neural development by PTBP1 and PTBP2. Nat Neurosc. 2012;15: 381-8.

27. Gudenas BL, Srivastava AK, Wang L. Integrative genomic analyses for identification and prioritization of long non-coding RNAs associated with autism. PLoS ONE. 2017;12:e0178532.

28. Yao RW, Wang Y, Chen LL. Cellular functions of long noncoding RNAs. Nat Cell Biol. 2019;21:542-51.

29. Tomikawa J, Shimokawa H, Uesaka M, Yamamoto N, Mori Y, Tsukamura H, et al. Single-stranded noncoding RNAs mediate local epigenetic alterations at gene promoters in rat cell lines. J Biol Chem. 2011;286:34788-99.

30. Gillberg C, Fernell E, Kocovska E, Minnis H, Bourgeron T, Thomson L, et al. The role of cholesterol metabolism and various steroid abnormalities in autism spectrum disorders: a hypothesis paper. Autism Res. 2017;10:1022-44.

31. Wang H. Lipid rafts: a signaling platform linking cholesterol metabolism to synaptic deficits in autism spectrum disorders. Front Behav Neurosci. 2014;8:104.

32. Fung LK, Libove RA, Phillips J, Haddad F, Hardan AY. Brief report: an open-label study of the neurosteroid pregnenolone in adults with autism spectrum disorder. J Autism Dev Disord. 2014;44:2971-7.

33. Sripada RK, Marx CE, King AP, Rampton JC, Ho SS, Liberzon I. Allopregnanolone elevations following pregnenolone administration are associated with enhanced activation of emotion regulation neurocircuits. Biol Psychiatry. 2013;73:1045-53.

34. Zhang J, Liu Q. Cholesterol metabolism and homeostasis in the brain. Protein Cell. 2015;6:254-64.
35. Compagnone NA, Mellon SH. Neurosteroids: biosynthesis and function of these novel neuromodulators. Front Neuroendocrinol. 2000;21:1-56.

36. Goritz C, Mauch DH, Pfrieger FW. Multiple mechanisms mediate cholesterol-induced synaptogenesis in a CNS neuron. Mol Cell Neurosci. 2005;29:190-201.

37. Spellman R, Llorian M, Smith CW. Crossregulation and functional redundancy between the splicing regulator PTB and its paralogs nPTB and ROD1. Mol Cell. 2007;27:420-34.

38. Anwar A, Abruzzo PM, Pasha S, Rajpoot K, Bolotta A, Ghezzo A, et al. Advanced glycation endproducts, dityrosine and arginine transporter dysfunction in autism - a source of biomarkers for clinical diagnosis. Mol Autism. 2018;9:3.

39. Castejon A, Spaw J. Autism and oxidative stress interventions: Impact on autistic behavior. Austin J Pharm Ther. 2014;2:1015.

40. Frye RE. Metabolic and mitochondrial disorders associated with epilepsy in children with autism spectrum disorder. Epilepsy Behav. 2015;47:147-57.

41. Page T, Coleman M. Purine metabolism abnormalities in a hyperuricosuric subclass of autism. Biochim Biophys Acta. 2000;1500:291-6.

42. Naviaux RK. Antipurinergic therapy for autism-An in-depth review. Mitochondrion. 2018;43:1-15.

43. Lee J, Cho YS, Jung H, Choi I. Pharmacological regulation of oxidative stress in stem cells. Oxid Med Cell Longev. 2018;2018:4081890.

44. Schneider L, Giordano S, Zelickson BR, S Johnson M, A Benavides G, Ouyang X, et al. Differentiation of SH-SY5Y cells to a neuronal phenotype changes cellular bioenergetics and the response to oxidative stress. Free Radic Biol Med. 2011;51:2007-17.

45. Doan RN, Bae BI, Cubelo B, Chang C, Hossain AA, AI-Saad S, et al. Mutations in human accelerated regions disrupt cognition and social behavior. Cell. 2016;167:341-54 e312. 